\title{
GEOMETRIC PROPERTIES, MINIMAX INEQUALITIES, AND FIXED POINT THEOREMS ON CONVEX SPACES
}

\author{
SEHIE PARK, JONG SOOK BAE, AND HOO KYUNG KANG
}

(Communicated by Palle E. T. Jorgensen)

\begin{abstract}
Using a selection theorem, we obtain a very general Ky Fan type geometric property of convex sets and apply it to the existence of maximizable quasiconcave functions, new minimax inequalities, and fixed point theorems for upper hemicontinuous multifunctions. Our results generalize works of $\mathrm{Ha}$, Fan, Jiang, Himmelberg, and many others.
\end{abstract}

\section{INTRODUCTION}

In 1961, Fan [F1] generalized the celebrated Knaster-Kuratowski-Mazurkiewicz theorem (simply, KKM theorem) and gave a number of applications in a sequence of his subsequent papers. For the literature, see [F2]. Fan [F1] also established an elementary but very basic "geometric" lemma which is equivalent to his $\mathrm{KKM}$ theorem. Later, Browder [Br] restated this result in the more convenient form of a fixed point theorem by means of the Brouwer fixed point theorem and the partition of unity argument. Now, it is well known that the Brouwer theorem, the Sperner lemma, the KKM theorem, Fan's geometric lemma, the Fan-Browder fixed point theorem, and many others are equivalent.

In many of his works in the KKM theory, Fan actually based his arguments mainly on the geometric property of convex sets. Since then there have appeared numerous applications, various generalizations, or equivalent formulations of Fan's geometric property or the Fan-Browder fixed point theorem.

The purpose in the present paper is to establish a very general Fan type geometric property of convex sets and to apply it to the existence of maximizable quasiconcave functions, new minimax inequalities, and fixed point theorems for upper hemicontinuous multifunctions. Our new geometric property generalizes that of $\mathrm{Ha}[\mathrm{Ha}$, Theorem 3]. His minimax inequality is also extended. Finally,

Received by the editors June 29, 1992; presented on November 7, 1992 at the 877 th meeting of the American Mathematical Society, University of Southern California, by the first author.

1991 Mathematics Subject Classification. Primary 47H10, 54H25; Secondary 49J35, 49K35, 52A07, 55M20.

Key words and phrases. Convex space, polytope, compactly closed, multifunction, KKM theorem, Tietze extension theorem, Urysohn's lemma, selection, partition of unity, upper semicontinuous (u.s.c.), acyclic map, quasiconcave, minimax theorem, upper hemicontinuous (u.h.c.), inward set.

Supported in part by KOSEF-GARC in 1992. 
for fixed point problems, we remove the paracompactness assumption in results of Fan [F2] and Jiang [J1, J2] and obtain a new Himmelberg type result for upper hemicontinuous multifunctions.

\section{SELECTION THEOREMS}

For the terminology and notation, we follow mainly Lassonde [L] and Park [P3, P4].

A convex space $X$ is a nonempty convex set in a vector space with any topology that induces the Euclidean topology on the convex hulls of its finite subsets. Such convex hulls will be called polytopes.

A subset $A$ of a topological space $X$ is said to be compactly closed (resp., open) in $X$ if, for each compact subset $K$ of $X$,the set $A \cap K$ is closed (resp., open) in $K$.

Multifunctions will be denoted by capital letters. For topological spaces $X$ and $Y$, a multifunction $G: X \rightarrow Y$ means that $G x$ is a nonempty subset of $Y$ for each $x \in X$.

Throughout this paper, a space means always a Hausdorff topological space.

We need the following:

Lemma 1.1. Let $L$ be a normal space, $K$ a compact subset of $L$, and $U$ an open subset of $L$. Suppose that $\alpha: K \rightarrow[0,1]$ is a continuous function satisfying $\operatorname{Supp} \alpha \subset U$. Then there exists a continuous extension $\tilde{\alpha}: L \rightarrow[0,1]$ of $\alpha$ satisfying Supp $\tilde{\alpha} \subset U$.

Proof. From the Tietze extension theorem, there exists a continuous extension $\alpha_{1}: L \rightarrow[0,1]$ of $\alpha$. Since $\operatorname{Supp} \alpha \subset U$, we can find an open set $V$ satisfying Supp $\alpha \subset V \subset \bar{V} \subset U$. Since $L$ is normal, by Urysohn's Lemma, there is a continuous function $\beta: L \rightarrow[0,1]$ satisfying $\beta(x)=1$ for $x \in \operatorname{Supp} \alpha$ and $\beta(x)=0$ for $x \in L \backslash V$. Then $\tilde{\alpha}=\alpha_{1} \cdot \beta$ is the desired extension of $\alpha$.

From Lemma 1.1, we have the following selection theorem:

Theorem 1.2. Let $X$ be a space, $Y$ a convex space, and $S, T: X \rightarrow Y$ multifunctions satisfying

(1.1) for each $x \in X, \varnothing \neq S x \subset T x$ and $T x$ is convex; and

(1.2) for each $y \in Y, S^{-1} y$ is compactly open in $X$.

Then, for any nonempty compact subset $K$ of $X$, there exists a continuous function $f: K \rightarrow Y$ such that

(1.3) $f x \in T x$ for each $x \in K$;

(1.4) $f(K)$ is contained in a polytope of $Y$; and

(1.5) for any compact subset $L$ of $X$ containing $K$, there exists a continuous extension $\tilde{f}: L \rightarrow Y$ of $f$ such that $\tilde{f} x \in T x$ for each $x \in L$ and $\tilde{f}(L)$ is contained in a polytope of $Y$.

Proof. Since $S x \neq \varnothing$ for each $x \in X$ by (1.1), $X$ is covered by $\left\{S^{-1} y: y \in\right.$ $Y\}$. Since $K$ is compact, $K \subset \bigcup_{i=1}^{n} S^{-1} y_{i}$ for some finite $\left\{y_{1}, y_{2}, \ldots, y_{n}\right\} \subset$ $Y$. Let $\left\{\alpha_{i}\right\}_{i=1}^{n}$ be a continuous partition of unity subordinated to the cover $\left\{S^{-1} y_{i} \cap K: 1 \leq i \leq n\right\}$ of $K$; that is,

(1.6) for each $i, \alpha_{i}: K \rightarrow[0,1]$ is continuous;

(1.7) Supp $\alpha_{i} \subset S^{-1} y_{i}$ for each $i$; and

(1.8) for each $x \in K, \sum_{i=1}^{n} \alpha_{i}(x)=1$. 
Now we define $f: K \rightarrow Y$ by $f x=\sum_{i=1}^{n} \alpha_{i}(x) y_{i}$ for $x \in K$. Then $f$ is a continuous function satisfying (1.4). Moreover, (1.1) implies (1.3).

In order to show (1.5), suppose that $L$ is a compact subset of $X$ containing $K$. Since $S^{-1} y_{i} \cap L$ is open in $L$ for $1 \leq i \leq n$, by Lemma 1.1, we have continuous extensions $\tilde{\alpha}_{i}: L \rightarrow[0,1]$ of $\alpha_{i}$ such that $\operatorname{Supp} \tilde{\alpha}_{i} \subset S^{-1} y_{i} \cap L$ for each $i$. Let $L^{\prime}=\left\{x \in L: \tilde{\alpha}_{i}(x)=0\right.$ for all $\left.i, 1 \leq i \leq n\right\}$. Then $L^{\prime}$ is compact and hence $L^{\prime} \subset \bigcup_{j=1}^{k} S^{-1} y_{n+j}$ for some finite $\left\{y_{n+1}, y_{n+2}, \ldots, y_{n+k}\right\} \subset Y$. Then $U_{n+j}=\left(S^{-1} y_{n+j} \cap L\right) \backslash K$ is an open subset of $L$ for each $j, 1 \leq j \leq k$, and $L^{\prime} \subset \bigcup_{j=1}^{k} U_{n+j}$ since $L^{\prime} \cap K=\varnothing$ by (1.8). Let $\left\{\alpha_{n+j}\right\}_{j=1}^{k}$ be a continuous partition of unity subordinated to the cover $\left\{U_{n+j}\right\}_{j=1}^{k}$ of $L^{\prime}$. Then, by Lemma 1.1 again, we have continuous extensions $\tilde{\alpha}_{n+j}: L \rightarrow[0,1]$ of $\alpha_{n+j}$ such that Supp $\tilde{\alpha}_{n+j} \subset U_{n+j}$ for each $j$. Note that $\tilde{\alpha}_{n+j}(x)=0$ for each $x \in K$ and each $j$ and that

$$
\sum_{i=1}^{n+k} \tilde{\alpha}_{i}(x) \neq 0 \text { for } x \in L .
$$

For each $i, 1 \leq i \leq n+k$, define $\beta_{i}: L \rightarrow[0,1]$ by

$$
\beta_{i}(x)=\frac{\tilde{\alpha}_{i}(x)}{\sum_{j=1}^{n+k} \tilde{\alpha}_{j}(x)} \quad \text { for } x \in L .
$$

Then each $\beta_{i}$ is a continuous function such that

(1.9) for each $i, \quad 1 \leq i \leq n+k, \operatorname{Supp} \beta_{i} \subset S^{-1} y_{i} \cap L$ and $\sum_{i=1}^{n+k} \beta_{i}(x)=1$ for $x \in L$; and

(1.10) for each $i, \quad 1 \leq i \leq n, \beta_{i}(x)=\tilde{\alpha}_{i}(x)=\alpha_{i}(x)$ for $x \in K$.

Now we define $\tilde{f}: L \rightarrow Y$ by

$$
\tilde{f} x=\sum_{i=1}^{n+k} \beta_{i}(x) y_{i} \quad \text { for } x \in L .
$$

Then, by (1.1) and (1.9), $\tilde{f} x \in T x$ for each $x \in L$, and by (1.10) we know that $\tilde{f}$ is a desired continuous extension of $f$. This completes our proof.

Remarks. 1. The merit of Theorem 1.2 is that the space $X$ may not be compact or paracompact and it enables us to extend several known theorems to nonparacompact cases.

2. Particular forms of Theorem 1.2 were due to Ben-El-Mechaiekh et al. [BDG1, Lemma (2); BDG2, Theorem 2.2] and Yannelis and Prabhakar [YP, Theorem 3.3], which are strengthened by Theorem 1.2.

\section{GEOMETRIC PROPERTIES OF CONVEX SPACES}

Recall that a nonempty space is acyclic if all of its reduced Čech homology groups over rationals vanish. In particular, any contractible space is acyclic, and thus any nonempty convex or star-shaped set is acyclic. For a space $Y$, $k a(Y)$ denotes the set of all compact acyclic subsets of $Y$. For a convex space $Y, k c(Y)$ denotes the set of all nonempty compact convex subsets of $Y$, and $c c(Y)$ all nonempty closed convex subsets.

For spaces $X$ and $Y$, a multifunction $F: X \rightarrow Y$ is said to be upper semicontinuous (u.s.c.) if $\{x \in X: F x \subset V\}$ is open for each open set $V \subset Y$. An u.s.c. multifunction $F: X \rightarrow k a(Y)$ is usually called an acyclic map. 
From the Lefschetz-type fixed point theorem for composites of acyclic maps due to Górniewicz and Granas [GG1, GG2], we have the following:

Lemma 2.1. Let $X$ be a compact space and $Y$ a convex space. If $f: X \rightarrow Y$ is a continuous function such that $f(X)$ is contained in a polytope $P$ and $F$ : $Y \rightarrow k a(X)$ is an u.s.c. multifunction, then $f F$ has a fixed point $y_{0} \in P$; that $i s, y_{0} \in(f F) y_{0}$.

Remark. Equivalent or particular forms of Lemma 2.1 appeared in Granas and Liu [GL], Ha [Ha], Komiya [K], Park [P4], and Shioji [Sh].

The following is a geometric property of convex spaces:

Theorem 2.2. Let $X$ be a space, $Y$ a convex space, and $B \subset C \subset X \times Y$ such that

(2.1) for each $x \in X,\{y \in Y:(x, y) \notin B\}$ is convex or empty;

(2.2) for each $y \in Y,\{x \in X:(x, y) \in C\}$ is compactly closed in $Y$; and

(2.3) for each $x \in X$, there exists a compactly closed subset $A_{x} \subset X \times Y$.

Further, suppose that there exists a nonempty compact subset $K$ of $X$ and, for each finite subset $N$ of $X$, there exists a compact subset $L_{N}$ of $X$ containing $N$ such that

(2.4) for each $y \in Y$,

$$
L_{N} \cap\left\{x \in X:(x, y) \in A_{z} \text { for all } z \in L_{N}\right\}
$$

is acyclic;

(2.5) for each $x \in L_{N} \backslash K$,

$$
\left\{y \in Y:(x, y) \in A_{z} \text { for all } z \in L_{N}\right\} \subset\{y \in Y:(x, y) \in B\} ;
$$

and

(2.6) for each $x \in K$,

$$
\left\{y \in Y:(x, y) \in A_{z} \text { for all } z \in X\right\} \subset\{y \in Y:(x, y) \in B\} .
$$

Then there exists a point $x_{0} \in X$ such that $\left\{x_{0}\right\} \times Y \subset C$.

Proof. Suppose that for each $x \in X$, there exists a $y \in Y$ such that $(x, y) \notin$ $C$. Define $S, T: X \rightarrow Y$ by

$$
S x=\{y \in Y:(x, y) \notin C\} \text { and } T x=\{y \in Y:(x, y) \notin B\}
$$

for $x \in X$. Then

(1.1) for each $x \in X, \varnothing \neq S x \subset T x$ and $T x$ is convex by (2.1); and

(1.2) for each $y \in Y, S^{-1} y=\{x \in X:(x, y) \notin C\}$ is compactly open in $X$ by (2.2).

Therefore, by Theorem 1.2, there is a continuous selection $f: K \rightarrow Y$ of $T$ satisfying (1.3)-(1.5). Now for each $x \in X$, let $K_{x}=\left\{z \in K:(z, f z) \in A_{x}\right\}$. Since $A_{x} \cap(K \times P)$ is closed in $K \times P$ by (2.3) and $f$ is continuous, $K_{x}$ is closed in $K$ for each $x \in X$.

We claim that $\left\{K_{x}: x \in X\right\}$ has the finite intersection property. Let $N$ be a finite subset of $X$ and $F: Y \rightarrow X$ defined by

$$
F y=L_{N} \cap\left\{x \in X:(x, y) \in A_{z} \text { for all } z \in L_{N}\right\}
$$

for $y \in Y$. Then each $F y \in k a\left(L_{N}\right)$ by (2.3) and (2.4).

Since $f$ satisfies (1.5), $f$ has a continuous extension $\tilde{f}: L_{N} \cup K \rightarrow Y$ such that $\tilde{f} x \in T x$ for $x \in L_{N} \cup K$ and $\tilde{f}\left(L_{N} \cup K\right)$ is contained in a polytope $P$ of 
$Y$. Since $L_{N}$ is compact, by (2.3), the graph of $F$ is closed and hence $F \mid P$ is u.s.c. Therefore, by Lemma $2.1, f F$ has a fixed point $y_{0} \in P$; that is, there is an $x_{0} \in L_{N}$ such that $x_{0} \in F y_{0}$ and $\tilde{f} x_{0}=y_{0}$. Since $(x, f x) \notin B$ for each $x \in X$ by the construction of $f$, we have $x_{0} \in K$ by (2.5), so that $\tilde{f} x_{0}=f x_{0}$. Hence $x_{0} \in(F f) x_{0}$, which implies $x_{0} \in \bigcap\left\{K_{x}: x \in N\right\}$ by the definitions of $F$ and $K_{x}$. This shows the finite intersection property of $\left\{K_{x}: x \in X\right\}$.

Since $K$ is compact, we have $\bigcap\left\{K_{x}: x \in X\right\} \neq \varnothing$. However, for any $z \in \bigcap\left\{K_{x}: x \in X\right\} \subset K$, we have

$$
(z, f z) \in \bigcap\left\{A_{x}: x \in X\right\}
$$

by the definition of $K_{x}$. Therefore, by (2.6), we have $(z, f z) \in B$, which contradicts the construction of $f$. Thus we must have an $x_{0} \in X$ such that $\left\{x_{0}\right\} \times Y \subset C$. This completes our proof.

Remarks. 1. In (2.3), compactly closedness of $A_{x}$ can be replaced by the following:

$(2.3)^{\prime} A_{x} \cap(L \times P)$ is closed in $L \times P$ for any compact subset $L$ of $X$ and any polytope $P$ in $Y$.

2. In Theorem 2.2, if $B=C$ and $A_{x}$ is a subset of $B$ independent to the choice of $x \in X$, then (2.5) and (2.6) hold automatically. In this case, Theorem 2.2 reduces to [Ha, Theorem 3$]$.

\section{EXISTENCE OF MAXIMIZABLE QUASICONCAVE FUNCTIONS}

In [Bl], Bellenger gave a theorem on the existence of maximizable quasiconcave real functions defined on paracompact convex spaces. His theorem is a common generalization of results of Fan [F2] and Simons [Sm1]. Bellenger also raised as an open question whether the paracompactness condition is necessary in his theorem. Park and Bae [PB] gave the affirmative answer to this question recently and applied it to obtain the Fan type nonseparation theorem and some coincidence and fixed point theorems. Those are refined and generalized in recent works of the first author [P2, P3].

In the present section, the geometric property in $\S 2$ is applied to give a more simple proof of a slightly different form of the existence theorem in [PB].

Recall that a real-valued function $f: X \rightarrow \mathbf{R}$ on a space $X$ is lower (resp., upper) semicontinuous (1.s.c.) (resp., u.s.c.) if $\{x \in X: f x>r\}$ (resp., $\{x \in$ $X: f x<r\}$ ) is open for each $r \in \mathbf{R}$. If $X$ is a convex set in a vector space, then $f$ is quasiconcave (resp., quasiconvex) provided that $\{x \in X: f x>r\}$ (resp., $\{x \in X: f x<r\}$ ) is convex for each $r \in \mathbf{R}$. Clearly, the quasiconvexity of $f$ implies that $\{x \in X: f x \leq r\}$ is convex for each $r \in \mathbf{R}$.

Theorem 3.1. Let $X$ be a convex space, $Y$ a convex space consisting of u.s.c. quasiconcave real functions on $X$, and $K$ a nonempty compact subset of $X$. Suppose that

(3.1) for each $x \in X, T x$ is a convex subset of $Y$ and $S x$ is a nonempty subset of $T x$;

(3.2) for each $f \in Y, S^{-1} f$ is compactly open in $X$; and

(3.3) for each finite subset $N$ of $X$, there exists an $L_{N} \in k c(X)$ containing $N$ such that, for each $x \in L_{N} \backslash K$ and each $f \in T x$, we have $f x<\max f\left(L_{N}\right)$.

Then there exists an $x \in K$ and an $f \in T x$ such that $f x=\max f(X)$. 
Proof. We put

$$
B=\{(x, f) \in X \times Y: f \notin T x\}, \quad C=\{(x, f) \in X \times Y: f \notin S x\},
$$

and, for each $x \in X$,

$$
A_{x}=\{(z, f) \in X \times Y: f z \geq f x\} .
$$

Then (3.1) and (3.2) imply (2.1) and (2.2), resp., and, for each $x \in X, A_{x} \cap$ $(L \times P)$ is closed as in $(2.3)^{\prime}$. Since each $f \in Y$ is quasiconcave and u.s.c., the set in (2.4) with $y=f$ is nonempty and convex. Hence (2.4) holds. It is easy to see that (3.3) implies (2.5).

Now suppose that the conclusion of Theorem 3.1 does not hold. Then, for each $x \in K$ and each $f \in T x$, we have $f x<\sup f(X)$; that is, for each $x \in K$ and each $f \in Y$ with $(x, f) \notin B$, we have $f x<f z$ for some $z \in X$ and hence $(x, f) \notin A_{z}$. Hence (2.6) also holds. Therefore, by Theorem 2.2, there exists an $x_{0} \in X$ such that $\left\{x_{0}\right\} \times Y \subset C$ and hence $S x_{0}=\varnothing$. This contradicts (3.1) and completes our proof.

If $T$ is single valued in Theorem 3.1, then we have the following:

Corollary 3.2. Let $f$ be an u.s.c. quasiconcave real function on a convex space $X$. Suppose that there is a nonempty compact subset $K$ of $X$ and, for each finite subset $N$ of $X$, there is an $L_{N} \in k c(X)$ containing $N$ such that

$$
f x<\max f\left(L_{N}\right) \text { for each } x \in L_{N} \backslash K .
$$

Then $\left\{x_{0} \in K: f x_{0}=\max f(X)\right\}$ is nonempty and compact in $K$.

Proof. By setting $Y=\{f\}$, we know that $Y$ is a convex space. Then the maximal set is nonempty by Theorem 3.1. Moreover, the set is the intersection

$$
\bigcap_{x \in X}\{y \in K: f y \geq f x\}
$$

of closed subsets of the compact set $K$.

Remark. Corollary 3.2 is essentially due to Park and Kim [PK] and extends earlier work of Mazur and Schauder. See also Park [P1].

\section{MinimaX THEOREMS}

In this section, we apply the geometric property in $\S 2$ to minimax theorems. The following generalizes Ha's minimax theorem [Ha]:

Theorem 4.1. Let $X$ and $Y$ be convex spaces and $f: X \times Y \rightarrow \mathbf{R}$ a l.s.c. function such that

(4.1) for each $x \in X, y \mapsto f(x, y)$ is quasiconcave on $Y$;

(4.2) for each $y \in Y, x \mapsto f(x, y)$ is quasiconvex on $X$; and

(4.3) there is a nonempty compact subset $K$ of $X$ and, for each finite subset $N$ of $X$, there is an $L_{N} \in k c(X)$ containing $N$ such that, for each $x \in L_{N} \backslash K$ and each $y \in Y$, we have

$$
\inf _{z \in L_{N}} f(z, y)<f(x, y) .
$$

Then we have

$$
\inf _{x \in X} \sup _{y \in Y} f(x, y)=\sup _{y \in Y} \inf _{x \in X} f(x, y) .
$$


Proof. Note that we always have

$$
\sup _{y \in Y} \inf _{x \in X} f(x, y) \leq \inf _{x \in X} \sup _{y \in Y} f(x, y) .
$$

We may assume that

$$
\sup _{y \in Y} \inf _{x \in X} f(x, y)=a<\infty
$$

without loss of generality. We define

$$
B=\{(x, y) \in X \times Y: f(x, y) \leq a\}
$$

and, for each $x \in X$,

$$
A_{x}=\{(z, y) \in X \times Y: f(z, y) \leq f(x, y)\} .
$$

Then we can easily check that all of the requirements $(2.1)-(2.6)$ in Theorem 2.2 hold with $B=C$. Therefore, there is a point $x_{0} \in X$ such that $\left\{x_{0}\right\} \times Y \subset B$. But this implies $f\left(x_{0}, y\right) \leq a$ for all $y \in Y$, and hence $\inf _{x \in X} \sup _{y \in Y} f(x, y) \leq a$. This completes our proof.

Remark. Even for the particular case $X$ and $Y$ are compact, Theorem 4.1 differs from Sion's minimax theorem [Si] in the continuity conditions. Instead of the lower semicontinuity of $f$ on $X \times Y$, Sion requires that, for each $x \in X$, $y \mapsto f(x, y)$ is u.s.c. and, for each $y \in Y, x \mapsto f(x, y)$ is l.s.c.

Simons [Sm2] gave a two-function version of Sion's theorem. Analogously, we can give a two-function version of Theorem 4.1 as follows:

Theorem 4.2. Let $X$ and $Y$ be convex spaces and $f, g: X \times Y \rightarrow \mathbf{R}$ such that $f \leq g$. Suppose that

(4.4) for each $x \in X, y \mapsto g(x, y)$ is quasiconcave and, for each $y \in Y$, $x \mapsto f(x, y)$ is l.s.c.;

(4.5) $g$ is l.s.c. on $X \times Y$ and, for each $y \in Y, x \mapsto f(x, y)$ is quasiconvex; and

(4.6) there is a nonempty compact subset $K$ of $X$ and, for each finite subset $N$ of $X$, there is an $L_{N} \in k c(X)$ containing $N$ such that, for each $x \in L_{N} \backslash K$ and each $y \in Y$,

$$
g(x, y) \leq \inf _{z \in L_{N}} g(z, y) \quad \text { implies } f(x, y) \leq \sup _{v \in Y} \inf _{u \in X} g(u, v) .
$$

Then we have

$$
\inf _{x \in X} \sup _{y \in Y} f(x, y) \leq \sup _{y \in Y} \inf _{x \in X} g(x, y) .
$$

Proof. We may assume that

$$
\sup _{y \in Y} \inf _{x \in X} g(x, y)=a<\infty .
$$

Define

$$
\begin{aligned}
& C=\{(x, y) \in X \times Y: f(x, y) \leq a\}, \\
& B=\{(x, y) \in X \times Y: g(x, y) \leq a\}
\end{aligned}
$$

and, for each $x \in X$,

$$
A_{x}=\{(z, y) \in X \times Y: g(z, y) \leq g(x, y)\} .
$$


Then clearly (4.4) implies (2.1) and (2.2). Also (4.5) implies that $A_{x}$ is closed in $X \times Y$ for each $x \in X$, and hence (2.3) holds. Since $L_{N}$ is compact, (2.4) and (2.5) hold by (4.6). Suppose that $z \in K$ and $(z, y) \in \bigcap\left\{B_{x}: x \in X\right\}$. Then we have $g(z, y) \leq g(x, y)$ for all $x \in X$, and hence

$$
f(z, y) \leq g(z, y) \leq \inf _{x \in X} g(x, y) \leq a .
$$

Therefore, $(z, y) \in B$ and condition (2.6) is satisfied.

Now we apply Theorem 2.2 to obtain an $x_{0} \in X$ such that $\left\{x_{0}\right\} \times Y \subset C$; that is, $f\left(x_{0}, y\right) \leq a$ for all $y \in Y$. Hence,

$$
\inf _{x \in X} \sup _{y \in Y} f(x, y) \leq f\left(x_{0}, y\right) \leq a .
$$

This completes our proof.

If $X$ is compact in Theorem 4.2, then (4.6) holds automatically. Hence, we have

Corollary 4.3. Let $X$ and $Y$ be convex spaces and $f, g: X \times Y \rightarrow \mathbf{R}$ functions satisfying (4.4), (4.5), and $f \leq g$. If $X$ is compact, then we have

$$
\min _{x \in X} \sup _{y \in Y} f(x, y) \leq \sup _{y \in Y} \min _{x \in X} g(x, y) .
$$

\section{FIXED POINT THEOREMS}

In this section, we apply the geometric property in $\S 2$ to fixed point problems.

Let $F: X \rightarrow E$ be a multifunction from a space $X$ with values in a real topological vector space $E$. We say that $F$ is upper demicontinuous (u.d.c.) on $X$ if, for an $x \in X$ and an open halfspace $H$ in $E$ containing $F x$, there exists a neighborhood $N$ of $x$ in $X$ such that $F y \subset H$ for all $y \in N$. Also we say that $F$ is upper hemicontinuous (u.h.c.) if, for each $f \in E^{*}$, the function $g x=\sup f(F x)$ is u.s.c. on $X$. It is well known that u.s.c. $\Longrightarrow$ u.d.c. $\Longrightarrow$ u.h.c.

Let $E$ be a vector space, $X \subset E$, and $x \in X$. The inward set $I_{X}(x)$ and outward set $O_{X}(x)$ of $X$ at $x$ are defined, resp., by

$$
\begin{aligned}
& I_{X}(x)=\{x+r(y-x): y \in X, \quad r \geq 0\}, \\
& O_{X}(x)=\{x+r(y-x): y \in X, \quad r \leq 0\} .
\end{aligned}
$$

A nonempty subset $L$ of a convex space $X$ is called a c-compact set if for each finite subset $N$ of $X, \overline{c o}(L \cup N) \in k c(X)$.

The following theorem extends results of Jiang [J1, J2], Fan [F2], and many others. For details, see Park [P3].

Theorem 5.1. Let $X$ be a convex subset of a real locally convex topological vector space $E$ and $F: X \rightarrow c c(E)$ an u.h.c. multifunction. Suppose that there exist a c-compact subset $L$ and a nonempty compact subset $K$ of $X$ such that

(5.1) for each $x \in K \cap \mathrm{Bd} X$ and each $f \in E^{*}$,

$$
\inf \left\{|f(y-z)|: y \in F x, \quad z \in I_{X}(x)\right\}=0 ;
$$

and

(5.2) for each $x \in \mathrm{Bd} X \backslash K$ and each $f \in E^{*}$,

$$
\inf \left\{|f(y-z)|: y \in F x, z \in I_{L}(x)\right\}=0 .
$$

Then $F$ has a fixed point. 
Proof. If $x \in \operatorname{Int} X$, then $I_{X}(x)=E$. Therefore, (5.1) can be stated as follows:

(5.1) for each $x \in K$ and each $f \in E^{*}$,

$$
\inf \left\{|f(y-z)|: y \in F x, \quad z \in I_{X}(x)\right\}=0 .
$$

We apply Theorem 2.2 with $B=C$. Let us define

$$
B=\left\{(x, f) \in X \times E^{*}: \inf f(F x) \leq f x\right\}
$$

and, for each $x \in X$,

$$
A_{x}=\left\{(z, f) \in X \times E^{*}: f z \geq f x\right\},
$$

where $E^{*}$ is endowed with any convex space topology. Then, (2.1) and (2.2) hold since $F$ is u.h.c. Now define $L_{N}=\overline{\operatorname{co}}(L \cup N)$ for any finite subset $N$ of $X$. Then $L_{N} \in k c(X)$ and, for each $f \in E^{*}$,

$$
L_{N} \cap\left\{x \in X: f x \geq f z \text { for all } z \in L_{N}\right\}
$$

is nonempty and convex. This shows (2.4). Moreover, (5.1) and (5.2) imply (2.6) and (2.5), respectively. Therefore, by Theorem 2.2 , there exists a point $x_{0} \in X$ such that $\left\{x_{0}\right\} \times E^{*} \subset B$. Since $E$ is locally convex and $F x_{0} \in c c(E)$, we can conclude that $x_{0} \in F x_{0}$. This completes our proof.

Remarks. 1. Note that (5.1) and (5.2) can be replaced by

$(5.1)^{\prime}$ for each $x \in K \cap \operatorname{Bd} X, F x \cap \overline{I_{X}(x)} \neq \varnothing$; and

$(5.2)^{\prime}$ for each $x \in X \backslash K, F x \cap \overline{I_{L}(x)} \neq \varnothing$, resp., without affecting the conclusion of Theorem 5.1.

2. Moreover, in Theorem 5.1, the inwardness conditions can be replaced by the corresponding outward conditions; that is, if we replace $I_{X}(x)$ and $I_{L}(x)$ by $O_{X}(x)$ and $O_{L}(x)$, resp., in (5.1) and (5.2) (and hence in $(5.1)^{\prime}$ and $\left.(5.2)^{\prime}\right)$, then Theorem 5.1 is still true.

Recall that Himmelberg [Hi] obtained a fixed point theorem for a compact u.s.c. multifunction $F: X \rightarrow k c(X)$, where $X$ is a convex subset of a locally convex topological vector space. Recently this was extended by the first author [P4] for $F: X \rightarrow k a(X)$. On the other hand, Deimling [D] proved a fixed point theorem for a condensing u.s.c. multifunction $F: X \rightarrow c c(E)$ which is weakly inward (that is, $F x \cap \overline{I_{X}(x)} \neq \varnothing$ for each $x \in X$ ), where $X \in c c(E)$ and bounded in a Banach space $E$.

Note that, from Deimling's theorem, in a Banach space, Himmelberg's result is extendable to a weakly inward multifunction. However, this is not known yet for locally convex topological vector spaces. We give a partial answer to this problem as follows:

Theorem 5.2. Let $E$ be a real locally convex topological vector space, $X \in c c(E)$, and $F: X \rightarrow c c(E)$ an u.h.c. multifunction. Suppose that there exist an $L \in$ $k c(E)$ and a nonempty compact subset $K$ of $X$ such that

(5.3) for each $x \in K, F x \cap \overline{I_{X}(x)} \neq \varnothing$; and

(5.4) for each $x \in X \backslash K, F x \cap L \cap I_{X}(x) \neq \varnothing$.

Then $F$ has a fixed point.

Proof. Define $B, A_{x} \subset X \times E^{*}$ as in the proof of Theorem 5.1. For each finite subset $N$ of $X$, we put $L_{N}=X \cap \overline{c o}(L \cup N)$. Then, as in the proof of Theorem 5.1, we can easily deduce (2.1)-(2.4) and (2.6) of Theorem 2.2 with $B=C$. 
In order to show (2.5), let $z \in L_{N} \backslash K$ and $f \in E^{*}$ such that $f z \geq f x$ for all $x \in L_{N}$. Since $F z \cap L \cap I_{X}(z) \neq \varnothing$ by (5.4), there exists a $y \in F z \cap L$ such that $(1-r) z+r y \in X$ for some $0<r \leq 1$. Since $(1-r) z+r y \in X \cap \operatorname{co}(L \cup\{z\}) \subset L_{N}$, we have $f z \geq f y$. Therefore, $(z, f) \in B$ and (2.5) holds.

Now applying Theorem 2.2, we have an $x_{0} \in X$ with $\left\{x_{0}\right\} \times E^{*} \subset B$, whence $x_{0}$ is a fixed point of $F$. This completes our proof.

Remarks. 1. If $L \subset X$, Theorem 5.2 is included in Theorem 5.1.

2. The inward set in Theorem 5.2 can not be replaced by the outward set even if $F$ is single valued:

Example. Let $f:[1, \infty) \rightarrow \mathbf{R}$ be given by $f x=0$ for all $x \geq 1$. Then $f$ satisfies conditions (5.3) and (5.4) for $X=[1, \infty), K=\{2\}, L=\{0\}$, and with $O_{X}(x)$ instead of $I_{X}(x)$. But $f$ has no fixed point.

As an immediate consequence of Theorem 5.2, we have the following:

Corollary 5.3. Let $E$ be a real locally convex topological vector space, $X \in$ $c c(E)$, and $F: X \rightarrow c c(E)$ an u.h.c. multifunction. Suppose that there exists an $L \in k c(E)$ such that, for each $x \in X, F x \cap I_{X}(x) \cap L \neq \varnothing$. Then $F$ has $a$ fixed point.

\section{REFERENCES}

[Bl] J. C. Bellenger, Existence of maximizable quasiconcave functions on paracompact convex spaces, J. Math. Anal. Appl. 123 (1987), 333-338.

[Bn] H. Ben-El-Mechaiekh, The coincidence problem for compositions of set-valued maps, Bull. Austral. Math. Soc. 41 (1990), 421-434.

[BDG1] H. Ben-El-Mechaiekh, P. Deguire, and A. Granas, Points fixes et coincidences pour les applications multivoques, C. R. Acad. Sci. Paris Sér. I Math. 295 (1982), 337-340.

[BDG2] _ Points fixes et coincidences pour les fonctions multivoques, II (Applications de type $\varphi$ et $\left.\varphi^{*}\right)$, C. R. Acad. Sci. Paris Sér. I Math. 295 (1982), 381-384.

[Br] F. E. Browder, A new generalization of the Schauder fixed point theorem, Math. Ann. 174 (1967), 285-290.

[D] K. Deimling, Fixed points of weakly inward multis, Nonlinear Anal. TMA 10 (1986), 1261-1262.

[F1] Ky Fan, A generalization of Tychonoff's fixed point theorem, Math. Ann. 142 (1961), 305-310.

[F2] _ Some properties of convex sets related to fixed point theorems, Math. Ann. 266 (1984), 519-537.

[GG1] L. Górniewicz and A. Granas, Some general theorems in coincidence theory. I, J. Math. Pures Appl. (9) 60 (1981), 361-373.

[GG2] _ Topology of morphisms and fixed point problems for set-valued maps, Fixed Point Theory and Applications (M. A. Théra and J.-B.Baillon, eds.), Longman Sci. Tech., Essex, 1991, pp. 173-191.

[GL] A. Granas and F.-C. Liu, Coincidences for set-valued maps and minimax inequalities, J. Math. Pures Appl. (9) 65 (1986), 119-148.

[Ha] C. W. Ha, Minimax and fixed point theorems, Math. Ann. 248 (1980), 73-77.

[Hi] C. J. Himmelberg, Fixed points of compact multifunctions, J. Math. Anal. Appl. 38 (1972), 205-207.

[J1] J. Jiang, Fixed point theorems for paracompact convex sets, Acta Math. Sinica 4 (1988), 64-71.

[J2] Fixed point theorems for paracompact convex sets. II, Acta Math. Sinica 4 (1988), 234-241. 
[K] H. Komiya, Coincidence theorems and saddle point theorem, Proc. Amer. Math. Soc. 96 (1986), 599-602.

[L] M. Lassonde, On the use of KKM multifunctions in fixed point theory and related topics, J. Math. Anal. Appl. 97 (1983), 151-201.

[P1] Sehie Park, Variational inequalities and extremal principles, J. Korean Math. Soc. 28 (1991), 45-56.

[P2] _ Applications of maximizable linear functionals on convex sets, Proc. Coll. Natur. Sci. Seoul Nat. Univ. 18 (1993), 23-33.

[P3] _ Fixed point theory of multifunctions in topological vector spaces, J. Korean Math. Soc. 29 (1992), 191-208.

[P4] - Some coincidence theorems on acyclic multifunctions and applications to KKM theory, Fixed Point Theory and Applications (K.-K. Tan, ed.), World Sci. Publ., River Edge, NJ, 1992, pp. 248-277.

[PB] Sehie Park and J. S. Bae, Existence of maximizable quasiconcave functions on convex spaces, J. Korean Math. Soc. 28 (1991), 285-292.

[PK] Sehie Park and S. K. Kim, On generalized extremal principles, Bull. Korean Math. Soc. 27 (1990), 49-52.

[Sh] N. Shioji, A further generalization of the Knaster-Kuratowski-Mazurkiewicz theorem, Proc. Amer. Math. Soc. 111 (1991), 187-195.

[Sm1] S. Simons, An existence theorem for quasiconcave functions with applications, Nonlinear Anal. TMA 10 (1986), 1133-1152.

[Sm2] _ Two-function minimax theorems and variational inequalities for functions on compact and noncompact sets, with some comments on fixed-point theorems, Proc. Sympos. Pure Math., vol. 45, part 2, Amer. Math. Soc., Providence, RI, 1986, pp. 377-392.

[Si] M. Sion, On general minimax theorems, Pacific J. Math. 8 (1958), 171-176.

[YP] N. C. Yannelis and N. Prabhakar, Existence of maximal elements and equilibria in linear topological spaces, J. Math. Economics 12 (1983), 233-245.

Department of Mathematics, Seoul National University, Seoul, 151-742, Korea

E-mail address: shpark@math.snu.ac.kr

Department of Mathematics, Myong-Ji University, Yongin, 449-800, Korea

Andong College of Technology, ANDong, 660, Korea 